\title{
Relaciones de Trabajo en el Contexto de la Globalización*
}

\author{
Suárez Picón, Cira *k \\ Márquez, Yasmiriam ** \\ Nava, Yuneska*** \\ Ojeda, América***
}

\section{Resumen}

El presente trabajo pretende esbozar los principales cambios en el mundo del trabajo, surgidos en el contexto de un nuevo orden mundial, influido por las variaciones del mercado y su integración global, así como por el impacto de las nuevas tecnologias; se refieren fundamentalmente a modificaciones expresadas en: la categorización de la fuerza laboral en trabajadores estables y contingentes, según la calidad de la relación contractual; la flexibilidad laboral en la contratación en función del número de trabajadores y el ajuste de la estructura salarial según productividad, así como el diseño de puestos multifuncionales; la desregulación del derecho del trabajo y la pérdida de capacidad reivindicativa de los trabajadores. Estos cambios conforman un entramado con otros aspectos variantes en la organización, de los cuales se derivan una serie de consecuencias que profundizan la precarización y la inestabilidad en las relaciones del trabajo. El estudio es producto de un análisis bibliográfico de tipo exploratorio sobre el tema, con algunas referencias empíricas correspondientes a la situación venezolana. Hasta ahora los resultados alertan sobre un mundo del trabajo con drásticos cambios que desmejoran al trabajador; pero a su vez, constítuyen retos fundamentales para una sociedad que aspire logros trascendentes, competitividad, productividad y equilibrio social.

Palabras clave: Relaciones laborales, globalización, organización del trabajo, flexibilización laboral, modernización empresarial.

Recibido: 01-04-26. Aceptado: 01-08-10

- Este trabajo forma parte del Programa Estrategias de Modernización Empresarial financiado por el Consejo de Desarrolio Científico y Humanistico de la Universidad del Zulia.

* Lic. en Administración, Profesoras de la Escuela de Administración e Investigadoras del Centro de Estudios de la Empresa (CEE) en la Facultad de Ciencias Económicas y Sociales (FCES) de la Universidad del Zulia (LUZ). E-mail: csuarez@iamnet.com; yasmiriamarquez@hotmail.com.

*** Lic. en Administración, adscritas al Programa Estrategias de Modernización Empresarial del CEE de la FCES de LUZ. 


\section{Work Relations Within the Context of Globalization}

\section{Abstract}

This paper intends to outline the principal changes in the area of work relations, which have occurred within the context of the new world order, influenced by market variations and global integration, as well as by the impact of new technologies. We refer fundamentally to modifications that have occurred in: the classification of workers as stable and contingent according to the quality of their contractual relation; labor flexibility in contracting based on the number or workers and the adjustment of the wage structure according to productivity and the design of multifunctional jobs; the de-regulation of the right to work, and the loss of replevin capacity on the part of workers. These changes constitute a framework, along with other aspects of organizational variants, from which derive a series of consequences which increase the precariousness and instability of work relations. This study is the product of an exploratory bibliographical analysis of the theme, with certain references to empirical situations in Venezuela. The results alert us to a world of drastic changes which will lower worker benefits, but which at the same time, will constitute fundamental challenges for a society that aspires to transcendent achievements, competitiveness, productivity and social equilibrium.

Key words: Labor relations, globalization, work organization, labor flexibility, enterprise modernization.

\section{Introducción}

En la década de los cincuenta el modelo Ohnista de organización del trabajo, desarrollado en la empresa Toyota en respuesta a la crisis interna y externa del mercado automotor japonés, inicia la conformación de un nuevo paradigma industrial compuesto por: un conjunto de innovaciones en la organización cuya importancia es comparable a lo que en su época fueron las innovaciones en la organización aportadas por el taylorismo y el fordismo (Coriat, 1995: 13). En la década de los setenta, los paises que habian alcanzado un mayor desarrollo industrial comienzan a cuestionar los costos de la producción en masa y como respuesta adoptan, entre otros, los postulados del modelo japonés. Con distintas características y dimensiones según cada caso, tanto la tecnología, como las estrategias de gestión empresarial en materia de organización del trabajo y empleo, van a sufrir múltiples transformaciones. En la década de los noventa, las estrategias de reconversión económica ligadas al aumento del intercambio comercial mundial se extendieron por todas las latitudes, influyendo sobre la vida social y política. Dichas estrategias forman parte de la Modernización Empresarial (Dombois $y$ Pries, 1993).

El proceso de Modernización introduce cambios en la organización del trabajo y genera modificaciones en las relaciones del trabajo expresadas en: nuevos lineamientos para la contratación del personal, modificaciones en el contenido de las calificaciones requeridas, ajustes en el número de trabajadores en la empresa según fluctuaciones de la demanda, re- 
ducción del tiempo de trabajo por la introducción de nuevas tecnologias, entre otras; que en un contexto de debilitamiento de la acción Sindical y del Derecho Laboral, profundizan la precarización y la inestabilidad en el trabajo. El estudio que se presenta implica un acercamiento a la realidad analizando y discutiendo ciertas referencias teóricas sobre el tema, así como el contexto de la globalización en el cual se profundizan estas estrategias internas de modernización impresarial.

\section{Un nuevo orden mundial: La globalización}

A fines del siglo $X X$ los avances simultáneos en campos como la informática, la biotecnología, la robótica, la microelectrónica, las telecomunicaciones han determinado nuevas posibilidades en la capacidad de generar productos y servicios con un horizonte de continuo crecimiento. Tal como to señala Callaghan (1997: 319): "El primer acontecimiento de importancia es la tecnología de la información en los últimos dos decenios. A medida que las telecomunicaciones y la informática adquirieron mayores niveles de sofisticación se acrecentó considerablemente la corriente de información entre zonas del planeta geográficamente distintas. Las empresas internacionales lograron descentralizar su producción en todo el mundo y mantener al mismo tiempo una estricta supervisión de sus actividades".

Este desarrollo que hemos presenciado, ha dado lugar al advenimiento de un nuevo modelo conocido como: Globalización o Mundialización. Según Villalonga (1996:1): "La Globalización es el pro- ceso por el cual la producción, distribución, y comercialización de un bien o servicio por parte de una empresa o grupo de empresas, se realiza de manera integrada por encima de las fronteras nacionales. Dicha Globalización se caracteriza porque la empresa proyecta su actividad sobre los mercados internacionales y lo hace de forma integrada, bajo una estrategia común".

En la actualidad, las empresas se encuentran en medio de un proceso de cambio, enmarcado dentro de la globalización, que según Yergin y Stanislaw (1999: 26) se ha convertido en una descripción más que familiar del proceso de integración e internacionalización de las actividades y estrategias económicas; éstos agregan que la misma no es un proceso sino una condición, un estado: una globalidad, una economía mundial en la cual las fronteras tradicionales y conocidas son desbordadas o se tornan irrelevantes.

Por su parte, Vargas (2000: 6) manifiesta que: "Teniendo en cuenta los acontecimientos económicos y políticos, la globalización consiste en un proceso colosal de concentración de capitales a nivel mundial. En él se opera la mayor liberalización para los movimientos de capital. Las naciones se ven condicionadas a ceder parte de sus potestades. La propia conducción macroeconómica y monetaria es vulnerada". De allí que un grupo importante de estrategias empresariales estén constituidas por fusiones, adquisiciones 0 absorciones.

Bassi (1999: 25) menciona que es un proceso de integración mundial que está ocurriendo en los sectores de comunicaciones, economía, finanzas y en los 
negocios, afectando por su amplitud y velocidad a los individuos, empresas y naciones; también señala Bassi que la caída de las barreras aduaneras, la formación de bloques económicos, la velocidad de las comunicaciones, los cambios tecnológicos y el flujo de capitales internacionales son las principales fuerzas que están moldeando un nuevo orden mundial. Este autor, lo mismo que Vargas (2000: 6), difiere de Yergin y Stanislaw (1999: 26) al catalogar la globalización como un proceso, pero a su vez concuerda con ellos al considerar que se basa en la integración mundial.

Ruiz (2000:1) define la globalización como un fenómeno que se gesta con la propia aparición del capital, por lo que su naturaleza es fundamentalmente económica, pero con repercusiones sociales, culturales, políticas, etc. En el contexto globalizado las empresas buscan aprovechar los diferentes tipos y estados de los mercados mundiales, para colocar su producción o capital (bancario, industrial o financiero) donde maximice su ganancia en las mejores condiciones y con la mayor libertad posible para su crecimiento y movilidad.

La globalización ocasiona un impacto en la integración de los mercados, la reorganización de las cadenas productivas y la integración de los flujos financieros; esto junto a las nuevas condiciones de competitividad impone un nuevo paradigma tecnológico (Rojas, 1999: 65-78) en materia gerencial especialmente laboral.

En las definiciones anteriores se puede observar que existe una concordancia entre los autores Vargas, Ruiz, Yergin y Stanislaw, y Villalonga al afirmar que la globalización se basa en la integra- ción internacional; por otra parte Vargas señala que la globalización permite el intercambio de bienes y factores de producción; Ruiz y Bassi expresan que tiene profundas repercusiones sociales, culturales y políticas; y por último concuerdan Ruiz y Vargas en que busca una mayor iibertad para el crecimiento y movilidad de la producción y del capital.

Desde la década de los sesenta, las extraordinarias transformaciones de la economia mundial han sido colectivamente denominadas Globalización o Mundialización. Para describir el perfeccionamiento de los patrones tradicionales de la actividad económica internacional y su expansión a nuevos ámbitos: tecnología, comercio, producción, finanzas, inversión e información; su alcance abarca nuevas regiones del mundo (Callaghan, 1997: 319).

Aunque los autores difieren en cuanto a si la globalización es una condición, un proceso o un fenómeno, para efectos de esta investigación la globalización es un proceso fundamentalmente de naturaleza económica que se caracteriza por disolver fronteras territoriales mediante la apertura comercial. Este proceso permite una relación más amplia con nuevos mercados para el intercambio de bienes y factores de producción, con la finalidad de lograr mejores niveles de competitividad, facilitando la movilidad de la producción y el capital, lo cual afecta a individuos, empresas y naciones.

Con la Globalización se hace indispensable aumentar la capacidad para responder rápida y eficientemente a las necesidades del mercado, dada la multiplicación constante de las interrelaciones entre los países. Uno de los objetivos que 
Relaciones de Trabajo en el contexto de la globalización

Suárez P., C.; Márquez, Y.; Nava, Y.; Ojeda, A.

persigue la globalización es conseguir economías de escala, en diversas áreas tales como: compra, logística, comunicaciones, entre otras. De esta forma la organización pretende crear ventajas competitivas en: calidad, productividad, y reducción de costos operativos; que le permitan un posicionamiento en el mercado local e internacional como estrategia viable de supervivencia.

Las organizaciones en su afán de reducir costos operativos modifican sus procesos de trabajo, racionalizándolos, incorporando mejoras tecnológicas, que transforman su estructura $e$ introducen una forma distinta de concebir la autoridad, el control y la toma de decisiones, desarrollándose un nuevo paradigma en lo que respecta al trabajo del hombre en la empresa y al conjunto de las relaciones laborales, lo cual afecta la distribución de sus tareas, permanencia o estabilidad, duración de la jornada laboral, flexibilización del sitio de trabajo, remuneración, capacitación y las relaciones sindicales entre otros.

Al respecto señala Callaghan (1997: 326): "Las economias desarrolladas lograrán recuperar su competitividad solamente cuando logren incrementar sus niveles de productividad. Esto implica: a) un esfuerzo de reestructuración del mercado de trabajo con miras a crear un sistema más flexible de prácticas laborales; b) un intento de mejorar las capacidades educativas, vocacionales y capacitación básica, particularmente en to que atañe al empleo de tecnologías nuevas".

La competitividad de la empresa en el mercado global se convierte en el fin prioritario y último al que se someten los demás, conformando un entramado de aspectos cambiantes en la organización, de los cuales se derivan una serie de consecuencias que profundizan la precarización, inequidad e inseguridad en el trabajo.

El proceso globalizador a pesar de sus bondades en materia tecnológica, de comercio e información comercial, producción, finanzas, inversión, presenta una serie de amenazas directas con respecto a los trabajadores, y para quienes se preocupan por la equidad social; dichas amenazas, corresponden a los tres dramas que describe Ermida (1999: 17-32):

a) Despreocupación relativa por el mercado interno: el modelo tradicional de sustitución de importaciones procuraba mantener un cierto poder adquisitivo de la población, ya que se producía para esos consumidores. Actualmente la preocupación se centra en el mercado externo globalizado, en el contexto de un sistema de libre comercio internacional; donde $\mathrm{ni}$ al gobierno ni a los empresarios les interesa ya el mercado interno y su poder adquisitivo, puesto que éste es cada vez menos su mercado.

b) El poder del Estado para dirigir el destino económico se ve disminuido y se derriban las barreras políticas para favorecer la libre competencia y el ingreso del capital extranjero; así el papel del gobierno nacional, como garante del Derecho Laboral y los Sindicatos, como fuerza política promotora de bienestar para el trabajador, quedan relegados bajo el interés del capital. El Derecho Laboral y la acción sindical son vistos como obstáculos al desarrollo de la libre empresa. 
c) Con el paradigma del interés económico por encima de los intereses laborales -la eficacia comercial justifica los medios- se introduce una nueva ideologia que no privilegia para nada los intereses del trabajador.

A las amenazas antes mencionadas se suman los efectos causados por la incorporación de la tecnología, respecto a la posibilidad de desplazar mano de obra, y es así como cada vez más, cada empresa termina por generar menos empleo que antes e incluso reduce su planta laboral.

Como señalan Montero $y$ otros (1999:11): "Frente a la magnitud de los cambios, las instituciones que se originaron y se acumularon a lo largo de la civilización industrial -regulaciones y normas diseñadas en su mayoría en fase de crecimiento y de conflicto agudo entre capital y trabajo- ya no están respondiendo a las nuevas necesidades. En momentos cuando emerge un nuevo paradigma productivo en el marco de una civilización post industrial, aún no hay consensos acerca del rol que debe jugar el Estado en la regulación del mercado de trabajo y las relaciones laborales. Tampoco está claro cómo deberia orientarse la renovación de los sindicatos".

\section{Cambios en el Mundo del Trabajo}

Las organizaciones a escala mundial diseñaron sus procesos con base en los postulados ideados por la administración científica y adecuados a un tipo de mercado masivo, homogéneo, que igualmente consolidó un modelo de relaciones laborales; luego con las transformaciones del mercada, de la demanda reducida de productos diferenciados, se ínició en Japón, especialmente en la empresa TOYOTA, un proceso de modernización que se ha venido difundiendo ampliamente.

Al respecto señala su creador, Taiichi Ohno citado por Coriat (1995:20): "EI sistema TOYOTA tuvo su origen en la necesidad particular en que se encontró Japón de producir pequeñas cantidades de muchos modelos de productos; más tarde, aquel evolucionó para convertirse en un verdadero sistema de producción. A causa de su origen, este sistema es fundamentalmente competitivo en la diversificación. Mientras el sistema clásico de producción, que planificaba en serie, es relativamente refractario al cambio, el sistema TOYOTA, por el contrario, resulta ser muy elástico; se adapta bien a las condiciones de diversificación más difíciles. $Y$ así es, porque fue concebido para ello".

El "sistema Toyota", o si se prefiere el Ohnismo, constituye un conjunto de innovaciones en la organización cuya importancia es comparable a lo que en su época fueron las innovaciones en la organización aportadas por el taylorismo y el fordismo. El ingeniero creador del método que revolucionó a la empresa Toyota en los años 1950 y siguientes, insiste siempre en dos puntos: El método Toyota es, la combinación de dos principios que considera sus pilares: la producción en el momento preciso y la auto activación de la producción. El resto, es cuestión de técnicas y de procedimientos. EI concepto de innovación se entiende como cualquier modificación de la organi- 
Relaciones de Trabajo en el contexto de la globalización

Suárez P., C.; Márquez, Y.; Nava, Y.; Ojeda, A.

zación que se traduzca en conceptos nuevos relativos a logística, planificación de las fabricaciones y asignación de tareas (Coriat, 1995: 13-14; 19-20).

Como parte de esas innovaciones señalan Dombois y Pries (1993: 16-18) las estrategias "blandas" de modernización: El control del trabajo por nuevas politicas de personal o tecnologías sociales que van más allá de la organización del proceso de trabajo, hacia la integración y la motivación intensiva de los trabajadores, tales como círculos de calidad para aprovechar los conocimientos de los trabajadores, promover la responsabilidad laboral e incrementar su integración a la empresa, desarrollando Identidad Corporativa.

Las innovaciones del Ohnismo conducen a modos de división del trabajo distintos de las formas clásicas de jerarquización y fraccionamiento de las tareas propias del taylorismo y el fordismo. En el proceso se configura la "fábrica mínima", reducida a las funciones, los equipos y el personal estrictamente requeridos para satisfacer la demanda diaria o semanal. El concepto de economía es indisoluble de la "reducción de los efectivos (personal)" y de la "reducción de costos" como condición esencial para la supervivencia y el crecimiento de un negocio (Coriat, 1995: 23).

La diversidad de cambios que se dieron en la empresa Toyota provocó serias reacciones de los sindicatos, por la intensificación del trabajo y sobre todo por la reducción de personal. Luego de sucesivas derrotas al sindicato, se consolidó el modelo de "relaciones industriales japonesas", cuyas características funda- mentales son: 1) sindicalismo de empresa 2) empleo de por vida, y 3) salario a la antigüedad. A esto se suman, favoreciendo la eficacia del sistema, los mercados internos de trabajo y los mecanismos sistemáticos de promoción, así como otras prestaciones a los trabajadores que, en definitiva, permitieron las transformaciones radicales introducidas por Ohno.

En tal sentido, Sotelo (1999: 100) señala: "Los nuevos métodos de organización del trabajo entramados en el paradigma toyotista, constituyen un instrumento para elevar la productividad y la cuota de ganancia y reorganizar el proceso de trabajo sobre otras bases, a partir de la descomposición del "obrero masa". Por lo menos esta es la dirección a que apunta el toyotismo, como paradigma que tiende a asumir un carácter universal, frente a la irreversibilidad del incremento de la precarización del trabajo, de la disminución cuantitativa y cualitativa del trabajo industrial, del crecimiento de los servicios y del "sector informal" en el seno de la larga onda depresiva de la economía capitalista mundial".

La introducción de estos nuevos métodos así como otras estrategias se ha denominado modernización. Modemizar, es transformar según las costumbres y gustos modernos. Modernizar constituye una aspiración de todas las épocas, para estar a tono con las circunstancias y exigencias del momento contemporáneo y adecuarse a algún modelo de referencia más avanzado - especialmente cuando se trata de los paises subdesarrollados por lo cual cuando se habla de modernización es necesario precisar su contenido; especialmente si se considera que 
existe un sin fin de interpretaciones acerca del concepto de modernización, siendo tal vez una de las constantes el uso de modelos del llamado mundo occidental.

Según Anita Weiss de Belalcázar citada por Dombois y Pries (Cf. 1993: 130): La Modernización no es simplemente el acercamiento a una meta o a un modelo predeterminado, sino un proceso abierto y permanente que, necesariamente, tiene un condicionamiento históri$\mathrm{co}$, cultural y social.

Según lo señalado por Dombois y Pries (1993: 13-14) se puede inferir como "Modernización Empresarial" al conjunto de cambios que se implementan en las empresas, para responder efectivamente a las condiciones imperantes. Al respecto, los autores refieren lo siguiente: "En los años ochenta comienzan en Europa y Latinoamérica, aunque aquí de una manera menos decidida y más selectiva, procesos de "reestructuración" del sistema de producción denominados "reconversión industrial" o "reestructuración". En las empresas se utilizan nuevas estrategias de tecnificación basadas en la microelectrónica, en nuevos conceptos de organización y en nuevas tecnologías sociales. No se trata únicamente de la continuación de líneas de racionalización o tecnificación tradicionales, sino de un cambio cualitativamente nuevo relacionado, sobre todo, con las condiciones básicas diferentes y las exigencias de los mercados, con los nuevos potenciales de tecnologias flexibles, con las nuevas estrategias gerenciales y con las nuevas políticas sindicales. Hablamos de la modernización de la producción empresarial, para denominar este complejo proceso de recientes cambios técnicos, organizativos y político-laborales en las empresas, que apuntan a diferentes metas como, por ejemplo, el incremento en la productividad, la calidad del producto y la flexibilidad productiva".

A partir de la afirmaciones anteriores sobre la Modernización, se evidencia que éste es un término con respecto al cual existen muy variados criterios; lo cierto en todo esto es que la misma, implica un cambio total de paradigma con respecto a cómo hacer las cosas, por lo tanto, está inevitablemente ligada al desarrollo económico, político y social. Sin embargo, el concepto de desarrollo también ha tenido diferentes interpretaciones, de la misma forma que han sido diversas las estrategias asumidas $0 \mathrm{im}$ puestas a los pueblos para lograrlo.

En consecuencia, el proceso de Modernización en el sector empresarial conlleva a una adaptación de éste a los grandes cambios que actualmente existen dentro de un mercado global para lograr mejores niveles de competitividad. Dentro de estos cambios, se destaca la Flexibilización del Trabajo, que puede asumirse en dos grandes vertientes: la relativa a la organización del trabajo y la que corresponde a las relaciones de trabajo.

La Flexibilización de la Organización del Trabajo, en nuestra opinión, se expresa en dos dimensiones:

a) Adaptación de los Procesos de Trabajo: para responder con facilidad a cambios en el volumen de la demanda y en el tipo de producto, según las exigencias del mercado, reajustando las etapas de cada proceso y la distri- 
Relaciones de Trabajo en el contexto de la globalización

Suárez P., C.; Márquez, Y.; Nava, Y.; Ojeda, A.

bución de máquinas y hombres. Esto incluye la automatización de las operaciones para disminuir tiempos de espera y costos. Paralelamente, se desarrolla al trabajador para cumplir una variedad de funciones en estos nuevos procesos, creando un trabajador polivalente o multihabilidoso.

b) Adaptación de la Estructura Organizacional: cambio de una estructura piramidal a una estructura horizontal o aplanada, reduciendo significativamente los niveles jerárquicos. Ante este tipo de estructura, surgen nuevos conceptos con respecto al control, autoridad, toma de decisiones, contenido del puesto, división del trabajo, entre otros.

Tal como se ha expresado, la "Modernización Empresarial" requiere de una nueva forma de organización de la producción y de gestión de la mano de obra; es evidente entonces que ello supone una adecuación de los mecanismos institucionales que regulan las relaciones entre trabajadores y empresarios, cuestión que discutimos en la siguientes sección, la cual constituye el núcleo de este trabajo.

\section{Un nuevo modelo de relaciones de trabajo}

En este contexto que se acaba de describir, el sistema tradicional de relaciones laborales viene presentando una serie de cambios de diversas formas. Es un entramado tal, en el que resulta difícil caracterizar cuáles cambios son responsabilidad directa de la globalización y cuáles no. Es importante portanto, destacar cómo era este sistema para poder en- tender la esencia del cambio que se viene operando y hacia donde se dirige.

El Sistema Tradicional que se está transformando tiene como fundamento una relación de trabajo en la cual el trabajador tiene un vínculo estable, por tiempo indeterminado, con el empleador. Es decir, el trabajador cuando es seleccionado y firma su contrato de trabajo, definido en términos de continuidad, se supone que se mantendrá en la empresa por un largo período, a menos que el trabajador o la empresa no cumplan con su parte, en cuyo caso la parte afectada da por terminada la relación. Por otro lado, el contenido del trabajo, es definido con claridad y son estables las tareas y responsabilidades, que sólo cambian con ocasión de un nuevo puesto. El trabajador que ingresa a la empresa lo hace para desarroilar una tarea especifica y como contrapartida recibe una remuneración esencialmente fija, con una jornada completa de trabajo, prevista en las Leyes (en el caso venezolano, ocho (8) horas laborales por dia), duración y pago del tiempo extra regulado, todo desarrollado dentro del espacio de la organización. Esa relación desde luego está resguardada por el Estado y por los Sindicatos, con su función tutelar del Derecho Laboral y la contratación colectiva, respectivamente.

Tal como señala William Bridges (1994: 15): "Hoy, el mundo del trabajo está cambiando una vez más. Las condiciones que crearon empleos hace 200 años, la producción en masa y la organización expandida, están desapareciendo... En lugar de empleos, ahora hay situaciones laborales temporales y a destajo. El cambio revela los síntomas de un 
cambio profundo que es más sutil pero a la vez más arraigado. El cambio profundo radica en que la organización de hoy se está transformando rápidamente de una estructura construida en base a empleos a un campo de trabajos que deben realizarse".

El trabajo asalariado desde finales del siglo XIX hasta mediados del siglo XX, se caracterizó por un sistema de producción en masa con puestos de trabajo rutinarios asignados a cadenas de producción, donde se hacía lo mismo una y otra vez; la noción del empleo en este contexto respondía a una estructura fija, predeterminada, regularmente rígida. Con el surgimiento de nuevos modelos centrados en la producción flexible y diversificada, cambia esta noción de empleo a otra referida a "campos de trabajos" para el desarrollo de procesos y no de tareas aisladas, las cuales se modifican según las exigencias del mercado.

Bridges (1994: 17) denomina a esta época como la era del "Post empleo" aludiendo al nuevo paradigma de las relaciones laborales, que junto a la Globalización y a los efectos del cambio tecnológico, se está delineando todavía.

A continuación se sistematizan algunas de las características que consideramos válidas para explicar el nuevo modelo de las relaciones del trabajo:

\subsection{Categorización de la fuerza laboral según la calidad de la relación contractual}

La tendencia en este nuevo orden mundial va hacia la transformación de las relaciones laborales por tiempo indeter- minado en relaciones temporales, flexjbles. Se reducen empleos y se utiliza personal temporal para llenar los espacios dejados o introducir el elemento de flexibilidad en la organización. Surge una fuerza laboral contingente, inestable, rotativa, informal que debe ser capaz de realizar diversas tareas de acuerdo a la necesidad organizacional, con una jornada laboral cambiante y reducida, ajustada a las variaciones de producción, tercerizada o subcontratada. Esta fuerza laboral coexiste con un grupo de trabajadores estables, en su mayoría con salarios elevados y alta calificación, con una duración más larga del contrato y desarrollo de carrera, para darle mayor seguridad en el trabajo.

Albizu (1997: 20-21) especifica las características del nuevo modelo de flexibilidad laboral que se erige en los últimos tiempos: "Podemos distinguir un número creciente de empleados centrales dedicados a las tareas relacionadas con el objeto social o core business, con derechos de seguridad y beneficios adicionales inherentes a sus puestos de trabajo. Este personal especializado se dedica a la empresa a tiempo completo, se halla dotado de un nivel de cualificación elevado y un alto compromiso con la compañía, y está disponible, con ciertos grados de flexibilidad, donde y cuando su trabajo lo requie$\mathrm{ra}$, y en la medida y capacidad necesaria... Para hacer frente a la demanda fluctuante y al crecimiento económico incierto, esta plantilla estaría apoyada por un personal periférico, de diversa condición, que aportaría flexibilidad numérica y financiera a la misma y tendría acceso a algunos de los beneficios y poco a la segu- 
Relaciones de Trabajo en el contexto de la globalización

Suárez P., C.; Márquez, Y.; Nava, Y.; Ojeda, A.

ridad en el empleo disfrutada por sus compañeros de la plantilla específica. Dentro del personal periférico, podemos distinguir, a su vez, entre el personal con una relación laboral no estable con la empresa (contratos de corta duración, contratos a tiempo parcial, puestos de trabajo compartidos, formación en la empresa) y el personal ajeno a la plantilla de la empresa que presta servicios en/para ésta (subcontrataciones con autónomos, subcontrataciones externas, subcontrataciones in situ)".

La empresa en la conquista de ventajas competitivas, que aseguren su integración global, perfila estrategias que profundizan la inequidad y la desigualdad de oportunidades en el ambiente de trabajo. Con respecto al personal, observamos dos clases de trabajadores: personal estable de primera categoría, que tiene una relación laboral estable con la empresa y personal contingente, cuya relación laboral es inestable, de acuerdo a la necesidad de producción, en función de la demanda y se contratan a tiempo parcial o temporal; aquí además se incluyen como estrategias, la conversión de los asalariados en microempresarios 0 cooperativas, la subcontratación de servicios especializados (Outsourcing) o tercerización y la subcontratación de suministradoras de mano de obra. Discrepamos del término "personal Periférico" utilizado por albiza, por cuanto los datos empíricos sugieren la contratación de tareas relacionadas con los procesos básicos (Core Business) en forma inestable.

Esta categorización aunque existen contraejemplos, no sólo se aplica en el sector empresarial. También se está imponiendo en el sector público, según se evidencia en los siguientes casos:

a) La Cervecería Polar C.A., modificó la relación laboral con los camioneros responsables de la distribución de sus productos, por contratos temporales renovables de distribución; fueron realizados con los antiguos trabajadores, bajo la figura de microempresarios, quienes debieron registrar sociedades mercantiles para distribuir de manera exclusiva cerveza y malta según condiciones impuestas por la Cervecería. Esta situación desencadenó un juicio, en reclamo de su condición de asalariados, que se ha prolongado cerca de diez años, con sentencias sucesivas a favor de los camioneros; en este momento se encuentra en la Sala Constitucional del Tribunal Supremo de Justicia para una decisión definitiva (Ver detalles en la sección 3.3 Desregulación del Derecho del Trabajo, Nota al pie No. 1).

b) En el sector público venezolano es habitual la contratación de suministradores de mano de obra para los senvicios de vigilancia y mantenimiento, tal como ocurre en universidades, hospitales, institutos autónomos y dependencias gubernamentales. Igualmente el Estado recurre a contratos con ONG's para prestar servicios públicos de capacitación, organización comunitaria, inserción laboral y escolar, etc.

c) El Hospital de Especialidades Pediátricas de Maracaibo (ente público), dispone de dos categorías de trabajadores. Un grupo que goza de cierta estabilidad, con contratos a tiempo determinado (un año de duración) en- 
tre quienes se encuentra el personal administrativo, médicos generales, enfermeras y bioanalistas. El resto de los trabajadores es personal contingente, al cual pertenecen: médicos especialistas, remunerados en función de su productividad (número de consultas, número de operaciones, etc.) y trabajadores suministrados a través de contratos con empresas especializadas en las áreas de: mantenimiento de equipos biomédicos, aire acondicionado, lavandería, limpieza, jardinería, servicio de alimentos, vigilancia, autoclave, calderas, ascensores y vigilancia.

d) En el caso de los Hoteles públicos y privados, como el Hotel del Lago y el Hotel Maruma, ambos categoría cinco estrellas, encontramos una situación donde se evidencian relaciones laborales estables, en contraposición con los casos anteriores. Paradójicamente, en estos Hoteles se conserva el sistema tradicional de relaciones del trabajo, con empleos a tiempo indeterminado y bajo relación de dependencia exclusiva con el Hotel. Según el criterio gerencial aplicado, este tipo de relación propicia en el empleado la conformación de una sólida cultura organizacional y el sentido de pertenencia; ambos son considerados fundamentales para ofrecer un servicio de calidad y reducir costos, condiciones que se dificultan con relaciones de trabajo bajo la modalidad de subcontratación, o con contratos inestables.

Previendo los serios efectos que enfrenta el "personal contingente" en la modalidad de subcontratación, Sotelo
(1999: 160) sentencia: "La Tercerización empresarial es una forma moderna de precarización del trabajo, en la medida en que fomenta y refuerza el proceso de pérdida de derechos de los trabajadores (eliminando importantes cláusulas en los contratos individuales y colectivos de trabajo) y coadyuva simultáneamente a reducir los costos de producción y a aumentar la tasa de ganancia. Algunos autores caracterizan la tercerización, o subcontratación, como un mecanismo propio de la "flexibilidad externa" que involucra a los trabajadores en la producción de las empresas contratantes, pero sin responsabilidad alguna para ellas".

\subsection{Flexibilidad Laboral}

Debido a la rotación necesaria de la mano de obra en un ambiente de globalización, como ya se ha señalado, el interés está puesto en la competitividad internacional; las organizaciones por tanto, piensan en reducir los costos del trabajo, es decir, reducir los puestos de trabajo y los beneficios laborales, a fin de abaratar los costos, como alternativa para aumentar las ganancias en la empresa. Sobre ésto se observa progresiva dada la ausencia de políticas coherentes por parte del Estado en su papel tutelar del trabajo.

Desde la óptica empresarial, se busca adecuar cualitativa y cuantitativamente el trabajo y los recursos, a la variabilidad de la demanda. Las organizaciones para el logro de este objetivo, en podrian contar con las siguientes estrategias: a) ajuste del número de trabajadores: consiste en la adecuación de los ingresos y despidos del personal, tanto 
Relaciones de Trabajo en el contexto de la globalización

Suárez P., C.; Márquez, Y.; Nava, Y.; Ojeda, A.

del grupo de trabajadores temporales como del estable. Las modalidades de flexibilización del despido que se aplican de hecho, originan la disminución de la planta de trabajadores permanentes de la empresa; b) diseño de puestos multifuncionales: consiste en la adecuación cualitativa de los trabajadores para adaptarlos a los cambios organizacionales; y c) ajuste de la estructura salarial: según el contenido del contrato (por obra, es decir, a destajo, por proyectos y contratos estables), la duración de la jornada y el establecimiento de sistemas salariales vinculados a la productividad.

En la flexibilización laboral, se pueden distinguir dos tipos de flexibilidad: la flexibilidad numérica-financiera y la flexibilidad funcional (Albizu, 1997:19). a) La flexibilidad numérico-financiera (inestabilidad del empleo): consiste en el ajuste continuo, al mínimo coste, del número de empleados - $u$ horas de trabajo contratadasque permitan a la empresa hacer frente rápidamente a las fluctuaciones de la demanda. Su carácter financiero se deriva de la variación de los costos laborales por el ajuste realizado sobre los contratos y las horas trabajadas, además el establecimiento de una estructura salarial relacionada con el desempeño individual o colectivo, y con los resultados obtenidos por la empresa. y b) La flexibilidad funcional (del cargo): es la capacidad de adecuar constantemente las competencias y tareas de los trabajadores a los cambios tecnológicos y a las exigencias de la demanda; es la polivalencia exigida por los modernos sistemas productivos. Los cambios que se producen en los productos, los servicios o los procesos productivos implican cam- bios de calificación laboral, tanto a corto como a mediano plazo.

La flexibilidad numérico-financiera o inestabilidad del empleo es la que predomina en Venezuela, especialmente en el sector privado aunque también existe en el sector público. Se puede evidenciar, por ejemplo, en las consecuencias de la decisión del gobierno venezolano, de monitorear el mercado petrolero, es decir, producir de acuerdo a las políticas de la Organización de Países Exportadores de Petróleo (OPEP) a fin de mantener 0 aumentar los precios del crudo. En el período 1998-1999 el gobierno de Hugo Chávez decidió reducir las cuotas de producción y aunque los precios del petróleo experimentaron una mejoría, por otro lado, se generaron despidos masivos que ocasionaron serios conflictos con los trabajadores petroleros. Esto sin contar con las empresas que dependen de este sector, como algunas empresas Metalmecánicas o aquellas que les suministran servicios, las cuales, ante la recesión del mercado petrolero, igualmente se ven afectadas, teniendo que ajustar la nómina de trabajadores a los vaivenes de la producción petrolera.

La capacitación del personal para facilitar la flexibilidad funcional o Polivalencia todavía no se ha generalizado. Sin embargo, se produce en algunos casos, sobre todo porque las organizaciones en la búsqueda del logro de sus fines y en su afán de ser competitivas, incorporan nuevas tecnologias o modifican sus procesos, lo cual conlleva a la necesaria capacitación de las personas que operarán estos nuevos equipos o desarrollarán los nuevos procesos. En el caso del Fondo 
Rotatorio para el Financiamiento de la Pequeña y Mediana Industria del Estado Zulia (FONFIDEZ) en el año de 1999, se implementó una Reingeniería de procesos y se adoptó un modelo de estructura plana, conformada por equipos de trabajo multifuncionales con responsabilidad colectiva sobre el resultado. El personal fue reubicado al asignarle la responsabilidad sobre los nuevos procesos y capacitado en las actividades a desarrollar para lograr los objetivos de la organización y reducir el tiempo total de trabajo.

Tanto la categorización, según la calidad del contrato de trabajo, como la flexibilidad laboral; propician relaciones de trabajo difusas en razón de un nuevo modelo de relaciones laborales, de manera tal que cada vez menos trabajadores están de hecho y legalmente habilitados para sindicalizarse y negociar colectivamente. El nuevo modelo de relaciones de trabajo se impone primero de hecho pero ejerce presiones para modificar las estipulaciones legales que protegen los derechos de los trabajadores. Estos dos aspectos se discuten a continuación.

\subsection{Desregulación del Derecho del Trabajo y Pérdida de Capacidad Reivindicativa}

Las relaciones laborales pasan de un marco colectivo a otro individual, donde cada trabajador negocia el precio de su trabajo en forma libre con su empleador; se transforma así, en materia de regulación privada entre las partes, lo que antes era competencia exclusiva de la Legislación Laboral. La actitud del Estado es la no intervención en las relaciones in- dividuales, mientras que en las relaciones colectivas, interviene a efectos de controlar la acción sindical, la negociación colectiva y el derecho a huelga, a fin de asegurar el libre juego de la oferta y la demanda de trabajo y salvaguardar los intereses del capital, lo cual se justifica, al menos "teóricamente" porque al abaratar los costos en materia laboral, se provocan nuevas inversiones, nuevos empleos (aunque precarios) y al final se lograría el beneficio social. En el ámbito de la negociación colectiva ha implicado un proceso de cambio que va de lo centralizado a negociaciones colectivas descentralizadas, perfilándose hacia lo individual. Así, según Albizu (1997: 18) se puede resumir esta acción flexibilizadora desde tres corrientes, bien diferenciadas:

- Desde el punto de vista sindical, la flexibilidad empresarial conduce a la flexibilidad laboral, que es una manera de reducir o suprimir derechos y conquistas sociales, así como las obligaciones legales que tienen las empresas en relación con toda una serie de cuestiones laborales que están normativamente reguladas, como son la jornada laboral, los horarios, los turnos, etc. Es decir, la flexibilidad laboral se identifica con la no regulación laboral.

- Desde el punto de vista patronal, la flexibilidad está habitualmente ligada con la necesidad percibida de eliminar todos los obstáculos legales al empleo de mano de obra, mediante la extensión o recuperación de prerrogativas de dirección de empresas. Además, la flexibilidad que pretenden muchos de los empresarios no se agota en la posibilidad de ajustar a la 
Relaciones de Trabajo en el contexto de la globalización

Suárez P., C.; Márquez, Y.; Nava, Y.; Ojeda, A.

baja las condiciones de empleo- en términos tanto cualitativos como cuantitativos- sino que abarca también la descentralización de los mecanismos reguladores.

- Desde el punto de vista de la administración pública, el acuciante problema del desempleo hace necesaria la intervención del Estado. En los últimos años, el aumento del desempleo, la disminución de las tasas de inversión y crecimiento, etc., evidencian la necesidad de cambiar las reglas del juego para adecuar las estructuras productivas a las necesidades de unos mercados cada vez más internacionalizados y competitivos. Asi, surgen medidas flexibles dentro del mercado laboral. Esta tendencia ha tenido su eco en España en la implementación de la denominada reforma del mercado de trabajo, plasmada en nuevas definiciones de la legislación laboral en lo concerniente al fomento de empleo, estatuto de los trabajadores, ley de procedimiento laboral, ley de sanciones y ley de agencias de colocación.

En el caso Venezolano, partir de los indicadores de ocupación y la composición del mercado Laboral, se puede evidenciar la necesidad de intervención del Estado a fin de reactivar el aparato productivo, y delinear políticas para la incorporación económica de los "sin empleo", para garantizar los derechos laborales que les son propios. Dichos indicadores son precisados por Zambrano (1999: 23): "La población ocupada totalizó 8.605.139 personas durante 1998 , de los cuales un $52 \%$ se encuentran ocupados en el sector formal de la economía mientras que el $48 \%$ restante se ubican en el sector informal. A pesar de que el sector formal registra salarios que son en promedio $42 \%$ superiores a los del sector informal, su capacidad de absorción de mano de obra crece a un promedio de $2.6 \%$ anual, creando un déficit de 5.7 puntos porcentuales con respecto a la tasa de crecimiento de la población económicamente activa; dicho déficit se tradujo, para 1998 en 253 mil personas que no encontraron empleo en el sector formal y por tanto, pasaron a engrosar las filas del desempleo abierto o se insertaron en el sector informal de la economía".

Por otra parte, según la Oficina Central de Estadística e Informática (OCEI) al cierre del primer semestre del 2000 , la tasa de desempleo se ubicó en $15.3 \%$, lo que significa que un millón quinientas cincuenta y cuatro mil seiscientas seis personas (1.554.606) se encontraban sin empleo. La cifra de personas del sector informal de la economía es la más alta de la última década: del total de personas ocupadas que suman ocho millones quinientos noventa y tres mil doscientos ochenta y nueve (8.593.289), el $52.6 \%$ forma parte del sector informal, mientras que $47.4 \%$ tienen empleos en el sector formal de la economia. Al permitir el estancamiento económico, la pérdida de empleo y la inflación, hemos diluido nuestro salario real. Ya en 1998 el salario real era la mitad del devengado en 1982 , o sea dieciséis años atrás. Dada la persistencia de la inflación a pesar de mantenerse en niveles inferiores a los de años anteriores, es fácil concluir que los salarios se han deteriorado aún más. 
Estas distorsiones del mercado de trabajo (aumento del desempleo y subempleo, desregulación, disminución del salario real, precarización) al igual que su impacto cultural y político, terminan por disminuir el poder de los trabajadores y sus representantes, para la legítima defensa de sus intereses.

En el devenir económico Latinoamericano, se ha venido observando el dominio de fórmulas individualizadas en las relaciones entre empresas y trabajadores, caracterizadas por el deterioro de las condiciones de trabajo y saláriales a lo largo de la región; Venezuela no es ajena a esta realidad y se vislumbra, aunque en menor proporción, el interés por flexibilizar las leyes en materia Laboral.

La reforma de la Ley Orgánica del Trabajo (L.O.T.) en 1997 incluyó novedades que favorecen el abaratamiento de las indemnizaciones por despidos, lo cual privilegia el empleo temporal, a saber: los artículos 108 y 146 , parágrafo segundo, donde se establece para efectos del cálculo de la antigüedad, su liquidación definitiva mensual con base en el salario devengado durante el mes en curso, lo cual eliminó el carácter retroactivo de las prestaciones de anti- güedad y cesantía, calculadas con base en el último salario.

Por otro lado, la L.O.T. no establece 10 relativo a las empresas de Trabajo Temporal (subcontratista u Outsourcing); sin embargo el Reglamento contempla esta modalidad en los artículos 23 al 28. El concepto de trabajador eventual u ocasional se expresa en el artículo 115 de la L.O.T, lo cual contribuye a formalizar las relaciones de trabajo flexibles y temporales. La práctica de contratar trabajadores bajo la apariencia de una relación mercantil con una microempresa, constituye un fraude laboral que se generaliza en el pais, pero que en algunos casos logra neutralizarse ${ }^{1}$.

Cabe destacar que la práctica laboral que se extiende en el sector privado y público es la de contrataciones a tiempo determinado por un máximo de 90 días con lapsos de interrupción de la relación laboral mayores de 30 dias, evadiendo así los patronos el pago de prestaciones sociales $y$ otros beneficios laborales, con base a lo establecido en los artículos 108 y 112 de la L.O.T. Esta situación constituye un desafío para el Ministerio del Trabajo, el cual no ha podido poner fin a esta ilegal práctica patronal.

1 La Sala Social del Tribunal Supremo de Justicia sentenció a favor de los trabajadores "microempresarios" contratados por la empresa Distribuidora Polar S.A, quienes debían registrar sociedades mercantiles para distribuir cerveza y malta, según condiciones impuestas por Cervecería Polar C.A, la cual buscaba evadir el pago de prestaciones sociales y otros beneficios de carácter laboral. La realidad identificada por el Juez era que dicha labor de compraventa debía ceñirse a condiciones impuestas por la Cervecería tales como: revender dichos productos a los detallistas indicados, no vender fuera de la zona de exclusividad, no vender productos de otras empresas, etc. El juez aplicó el Art. 65 L.O.T. el cual define la existencia de una relación de trabajo de hecho. Al cierre del informe de PROVEA, la empresa impugnó ante la sala Constitucional, creándose gran expectativa por la decisión final que tendrá este caso tan importante para las relaciones obrero-patronales (XII Informe Anual de los Derechos Humanos en Venezuela. PROVEA). 
Relaciones de Trabajo en el contexto de la globalización

Suárez P., C.; Márquez, Y.; Nava, Y.; Ojeda, A.

En el país, se privilegia la existencia de sindicatos de empresa; la L.O.T. determina los requisitos para su conformación, y establece en el artículo 417, un mínimo de veinte (20) trabajadores para constituir un sindicato de empresa, no obstante, este número suele ser superior con relación al tamaño promedio de las empresas. Además, se privilegia la negociación colectiva descentralizada, lo que al final disminuye el poder de negociación y limita sus resultados. Adicionalmente, los sindicatos en la actualidad afrontan un profundo cuestionamiento a su papel y práctica tradicional y se promueve, por parte del Gobierno, la creación de una central única de trabajadores pese a lo establecido en materia de libertad sindical en el título tercero del reglamento de la L.O.T. (artículos 142 y siguientes). Venezuela, el 10-08-78 se suscribió al Pacto Internacional de Derechos Económicos, Sociales y Culturales (Pidesc) que establece en su artículo 8 el Derecho a la Libertad Sindical; además, ha ratificado 56 convenios de la O.I.T., incluyendo en Junio de 1998, la Declaración sobre los Derechos Humanos Laborales. Por otra parte, el artículo 3 del Convenio 87 de la O.I.T. establece que los sindicatos tienen derecho a elegir libremente a sus representantes... y que "las autoridades públicas deberán abstenerse de toda intervención que tienda a limitar este derecho o a entorpecer su ejercicio legal". El Gobierno, por tanto, no debería intervenir en el funcionamiento interno de las organizaciones sindicales, ni decidir cuando se efectuarán elecciones en su seno y menos aún decidir sobre su unificación.
Es importante destacar que el trabajo y la remuneración salarial son esenciales en una sociedad. El trabajo es la fuente de ingreso del trabajador y su familia; sin trabajo no hay ingreso y por tanto, disminuye sustancialmente el poder de compra. Se puede decir que sin salario, el ciudadano como miembro de la nación y la Sociedad, es un inválido política y socialmente; de allí que el Estado en su fin social, tenga la responsabilidad de protegerlo y regularlo. No obstante, en el actual contexto globalizador, los lineamientos son otros, las reglas del juego cambian como se ha señalado anteriormente.

\subsection{Nuevas tecnologías: reducción del tiempo de trabajo $y$ desplazamiento de mano de obra}

En la búsqueda de la productividad, la calidad y la competitividad, la empresa incorpora cambios tecnológicos para responder más eficientemente a las necesidades del mercado globalizado. EI progreso Técnico ha llevado desde sus inicios a una constante reducción del tiempo de trabajo, provocando el desplazamiento de mano de obra por la introducción de equipos y nuevas tecnologías. Albizu (1997: 7-8) analizando este aspecto, alerta sobre la velocidad del cambio tecnológico, tanto de la información como de la producción, y su globalización, así como sus repercusiones: "EI avance tecnológico genera modificaciones sustanciales en los sistemas de producción, así como en la forma de trabajo y de vida. En especial, la informática, y otras tecnologías afines han sufrido un 
desarrollo sin precedentes en la historia, que se caracteriza por la gran rapidez de sus innovaciones, la universalidad de sus aplicaciones, la facilidad de difusión, las sinergias que se generan en torno a ellas (cualquier avance en una de ellas repercute rápidamente en el grupo tecnológi$c o$ ), la progresiva sustitución de personas por máquinas, y los cambios estructurales que generan en las empresas". Rifkin (1998) afirma que El trabajador más barato del mundo, no será tan barato como la tecnología que va a reemplazarlo"

Según un estudio realizado en las empresas metalmecánicas del Municipio Maracaibo (Fuenmayor, 2000: 4445):.. "el $75 \%$ de los gerentes de recursos humanos entrevistados afirmaron que en los próximos años no contratarán secretarias, recepcionistas y operadores, sencillamente porque se ubicarán en el área de empleos marginales innecesarios, sustituidos por el mundo de la informática; el personal semicalificado sólo será contratado por pequeñas empresas con escasa tecnología. Estos mismos gerentes coincidieron en afirmar que un aumento significativo de la producción en estas empresas no implicará contratación de mano de obra; contrariamente, la mayoría afirmó que las empresas venezolanas disminuirán la nómina de trabajadores y reducirán los turnos de trabajo, consecuencia lógica de la necesidad de estar en sintonía con los cambios tecnológicos que se producen en el mundo".

Sotelo (1999: 87) señala que los "cambios en la composición del trabajo producidos por las políticas de desindustrialización neoliberal en el mundo, han desplazado al trabajo vivo por la maqui- naria y la tecnología, introducido el predominio de los servicios sobre el trabajo industrial y los nuevos métodos de organización del trabajo, la informalidad y la precarización".

El nuevo modelo de relaciones de trabajo, profundiza el auge del desempleo y aumenta la brecha de desigualdad e inseguridad social, disminuyendo la calidad de vida de los ciudadanos. En este orden de ideas, Enrique Iglesias presidente del Banco interamericano de Desarrollo (B.I.D.) ha manifestado (1999: 14): "América Latina es la región con mayor grado de inequidad en el mundo; tiene la mayor desigualdad en la distribución de los ingresos, 10 millones de personas desempleadas, la mayoría mujeres o menores de 25 años. La brecha salarial entre trabajadores calificados y no calificados es la más alta del mundo. La región tiene que reformar el sistema laboral, mejorar la educación y promover el desarrollo del sector informal. Un $56 \%$ de la fuerza laboral en América Latina trabaja en el sector informal, donde 50 millones de pequeñas o microempresas son el principal generador de empleos".

De esta manera la Reducción del tiempo de trabajo, el desplazamiento de mano de obra por introducción de nuevas tecnologías, sumados a la categorización de la fuerza laboral según la calidad de la relación contractual, la flexibilización laboral y la desregulación del derecho del trabajo, describen el perfil de las actuales relaciones del trabajo tanto para el sector privado, como para el sector público; y cada vez son más influidas por el proceso de la globalización que tiende a universalizar los modelos y estrategias empresariales. 


\section{Conclusiones}

El término globalización es utilizado en distintos sentidos, aunque pueden mencionarse elementos comunes a todas las versiones, tales como: 1) El centro de interés de la globalización es eco. nómico y tecnológico, en las áreas de producción, finanzas, comercio, inversión, senvicios e información 2) La globalización elimina fronteras mediante la interconexión de las economías, especialmente en lo relacionado con el mercado (integración global) 3) La globalización genera efectos sociales, culturales y politicos.

En razón de la integración global, la necesidad de captar nuevos mercados y los avances tecnológicos, se han operado cambios fundamentales en la organización del trabajo empresarial, privado y público, que forman parte de la Modernización Empresarial, y persiguen básicamente la reducción de costos, pero también aumento de la diversidad y calidad del producto, entre otros, a fin de lograr la competitividad.

Los cambios en la Organización del Trabajo, especialmente la flexibilidad productiva, imponen modificaciones en la calificación y competencias de los Recursos Humanos, a fin de adecuarlos al nuevo contenido del trabajo, definición de puesto, interdependencia de funciones y roles que aumentan la polifuncionalidad 0 multihabilidad.

Las estrategias empresariales incorporadas a raíz de la modernización empresarial conforman un nuevo paradigma de relaciones del trabajo caracterizado por: categorización de trabaja- dores (estables y contingentes); rotación laboral, multifuncionalidad, ajuste de las estructuras saláriales; desregulación o flexibilización del Derecho Laboral, pérdida de la capacidad reivindicativa de los trabajadores y reducción del tiempo de trabajo necesario para producir bienes $y$ servicios, con el consecuente desplazamiento de mano de obra. Todo lo anterior profundiza la precarización o desmejoramiento de las condiciones del trabajo.

\section{Bibliografía citada}

Albizu, Eneka (1997). Flexibilidad Laboral y Gestión de los Recursos Humanos. Ariel Sociedad Económica. España. P. 186.

Bassi, Eduardo (1999). Globalización de Negocios. Construyendo Estrategias Competitivas. Editorial LIMUSA;S.A. México.

Bridges, William (1994). Fortune Magazine. The time Inc. P.14-21.

Callaghan, Lord (1997). Informe sobre las conclusiones y recomendaciones del grupo de expertos de alto nivel sobre oportunidades y riesgos de la Globalización. El Foro Internacional, Vol. XXXVII. No. 2. P. 319-328.

Congreso de la República de Venezuela. Ley Orgánica del Trabajo (1997) y su Reglamento (1999).

Coriat, Benjamin (1995). Pensar al Revés: Trabajo y Organización en la Empresa Japonesa. México. Editores, S.A. Siglo XXI. P. 161.

Dombois, Rainer y Pries, Ludger (1993). Modernización Empresarial. Tendencias en América Latina y Europa, Ediciones Nueva Sociedad, Colombia.

Ermida, Oscar (1999). Las Relaciones de Trabajo en el siglo XXI. III Congreso Regional de las Américas. Asociación 
Peruana de Relaciones de Trabajo (APERT). P. 17-32.

Fuenmayor, Nila y Gamboa Cáceres, Teresa. (2001). ¿Empleo juvenil o reproducción de la pobreza en Venezuela? Documento inédito. CEE - Maracaibo, Venezuela.

Iglesias, Enrique (1999) Informe del Banco Interamericano de Desarrollo (B.I.D.). P.14.

Montero Cecilia, Alburqueque Mario y Jaime Ensignia (1999). Recolocando el Trabajo en el Debate. Trabajo y Empresa entre dos siglos. Editorial Nueva Sociedad Venezuela. P. 9-15. OCEI PROVEA ( ) XII Informe Anual de los Derechos Humanos en Venezuela.

Oficina Central de Estadística e Informática OCEI (2000). Indicadores de la Fuerza de Trabajo (cierre del primer semestre). P. 22.

Organización Intemacional del Trabajo O.I.T. Normas Intemacionales del Trabajo. Convenio 87: "Libertad Sindical y Protección del derecho de sindicación", 1948. Pág. Web: ilolex.ilo.ch:1567/cgilex/convds.pl?CO 87. pp.2.Consulta: 07.08.01.

PROVEA (2000). XII Informe Anual de los Derechos Humanos en Venezuela. Situación de los Derechos Económicos, Sociales y Culturales. http://www.derechos.org.ve/ 30/04/01.
Rifkin, Jeremy (1998). El fin del trabajo. Conferencia grabada. Caracas.

Rojas, Claudio (1999). "Internacionalización y acción pública. La complejización de la organización del trabajo en la estrategia de desarrollo chilena". Trabajo y Empresa: entre dos siglos. Editorial Nueva Sociedad. P. 65-78.

Ruiz, Verónica (2000). Discurso de la Globalización. http://www.reci.net/globalizacion/lega/fg109.htm pp7; 02/07/00.

Sotelo, Adrián (1999). "Globalización y Precariedad del trabajo en México". Ediciones el Caballito, S.A. México. P.191.

Vargas T. Martha (2000). Globalización, Desarrollo Regional y Atomización del Estado Nación. http: //members.tripod.com.co/global/146actualproceso2.htm pp6 02/07/00.

Villalonga, Magda (1996). La Globalización de los Mercados en la Distribución. Pág web:www.aecoc.es/, documentos de hemeroteca AECOC. 7 pp. Consulta 11-07-00.

Yergin, Daniel y Stanislaw, Joseph (1999). Pioneros y Líderes de la Globalización. Javier Vergara Editor. Buenos AiresArgentina.

Zambrano, Sequín Luis (1999). Política Económica, Estabilización y Pobreza: La situación macroecónomica en 1999. 